\title{
"Purpose" in a cognitive theory of reference
}

\author{
JOHN M. CARROLL \\ IBM Watson Research Center, Yorktown Heights, New York 10598
}

\begin{abstract}
Two experiments examined the creation of name expressions for reference, with specific regard to Olson's (1970) "cognitive theory of semantics." In the first experiment, subjects were merely asked to make up names for each of a series of abstract symbols embedded in displays. In the second experiment, subjects were asked to make up names in order to verbally distinguish between symbols in each display. Only under the second procedure were the predictions of Olson's analysis confirmed. These results suggest that naming, and therefore reference in general, cannot be comprehensively treated in a theory that fails to explicitly deal with referential purposes.
\end{abstract}

Olson (1970) proposed a simple and bold cognitive analysis of reference. Referring, in this view, is equivalent to distinguishing an entity from its relevant context. Olson operationalized this claim in a series of demonstrations: Imagine that a gold star has been placed under a small, round, white wooden block. One participant in the demonstration is aware of this; another is not. The target block is now presented to both participants, and the one who knows where the star is must tell the other one where to look for it. Various other blocks are sometimes presented with the target (for example, a small, black, round block). The participant that knows where the star is might say, "The star is under the white one," referring to the target block with only the minimal differentiating label. (This type of referential situation has also been analyzed by Brown, 1965, p. 265; Carroll, in press-b; Krauss \& Weinheimer, 1967; Rosenberg \& Cohen, 1964.)

Since the context set, that is, the nontarget blocks, systematically influenced what sort of name expression was employed in the task, Olson (1970) concluded that names do not designate referents, but rather they distinguish referents from alternatives. He described this as the "paradigm case" of reference; we shall refer to it as the "paradigm case naming strategy," or PCS. Olson's conclusion, however, is not warranted by the demonstration. The "referential" character of Olson's task situation was confounded with the implicit task

The title of this article intentionally recalls the title of Olson (1970). However, whereas Olson seemed to confuse the terms "semantics" and "reference," I have correctly used only the latter (see Carroll, Note 1). Requests for reprints should be directed to the author at 11-105, T. J. Watson Research Center, Yorktown Heights, New York 10598. I am grateful to Carol-Jo Chiero, Stewart Flythe, and Fran Milite for help in analyzing the data presented here, to the members of the New York Psycholinguistic Circle for comments on an oral presentation of this research, and to John T. Richards for comments on an earlier version of the manuscript. demand to distinguish targets from nontargets. Two replications of Olson's demonstrations were conducted to isolate the role of "purpose" in reference.

\section{EXPERIMENT 1}

The experiment was embedded within an extensive study of naming (Carroll, 1980, in press-a, Note 2), in which subjects created names for various textual and graphic entities. It was hoped that this would make implicit task demands less salient. The relevant stimulus set consisted of symbols taken from Dreyfus (1972).

\section{METHOD}

Sets of three symbols each were pasted onto $244 \times 6$ in. index cards. An arrow pointed to one symbol, the target. The remaining two symbols (the nontargets) bore one of four relationships to the target symbol. These relationships were defined as follows. Full relational-All three of the symbols on the card were identical. Mixed relational-Two of the three symbols on the card were identical. One of these two was the target. Homogeneous attributive-All of the three symbols were distinct, but all three were relatively similar, having been drawn from a common subsection in Dreyfus (1972). Heterogeneous attributive-All of the three symbols were distinct, and none were drawn from a common subsection in Dreyfus (1972). The entire set of materials appears as Appendix 1 of Carroll (Note 2).

Ten subjects were run either singly or in pairs. Instructions to subjects consisted of a general statement about naming and reference, followed by a description of the experimental task (these instructions appear as Appendix 1 of Carroll, 1980). In addition to this general material and instruction, the following direction appeared on a cover card to the symbol-triples portion of the experiment: "On each card there will be three graphic symbols. An arrow will point to one of them. By what name would you call the symbol pointed to?" In the instructions and in answers to subjects' questions, the experimenter avoided defining or delimiting what could count as a name. This responsibility was, insofar as possible, delegated entirely to the subject.

Subjects examined each of the 24 symbol displays in sequence. Half of the subjects received one blocked presentation sequence, and the other half received the reverse sequence. For each display, subjects first recorded a name for the target symbol 
and then rated the name on two 1-7 scales: goodness and ease of creation. Good names were defined to be names that are easy to learn and remember (descriptive, natural, etc.) and easy to use (distinctive, brief, etc.). Ease referred to how difficult it was for the subject to generate the name in the first place. In both cases, a rating of 7 designated the labeled extreme of the scale.

\section{RESULTS AND DISCUSSION}

Olson's (1970) analysis of reference makes two predictions here. Since in the case of the full relational displays the only differentiating feature of the target is position, Olson would predict "position word" names: "left," "right," "middle," and so on, or possibly "the left one," "the right object," and so on. This tendency should be relaxed somewhat in the mixed relational display condition, since in this case only one nontarget is structurally identical to the target. Here, perhaps, names would include some structural information (e.g., the left one with ears, the middle circle shape, etc.). Both of the relational display conditions should follow the predictions of the PCS more closely than either of the attributive display conditions, which can in this sense be thought of as control conditions.

Assuming, however, that these two predictions were confirmed, the present experiment affords a further elaboration of the PCS. Consider the difference between the homogeneous and heterogeneous attributive display types. In the former type, the three symbols composing the display are more structurally similar than in the latter type. In such a case, an "extended" PCS might predict that more position words should occur in names rendered for homogeneous than for heterogeneous displays.

The primary dependent variable was the frequency of name expressions consisting wholly of positional words, across the four stimulus conditions. Positional words were defined by the class first, second, last, center, central, middle, right, and left. If a name expression consisted only of such a position word and optionally an "empty" noun, such as "one," it was scored as a purely "position word name." Empty nouns consisted of the class object, thing, shape, one, symbol, character, pattern, and design. Examples of purely position word names, from the data, included "left character," "left-hand," "symbol on the left," and so on.

It is clear by mere inspection of the summary data in Table 1 that Olson's (1970) PCS was not the strategy of choice in the present experimental situation. It accounts for less than $4 \%$ of all the names rendered. However, a more sensitive assessment of the strategy can be made by contrasting the relative frequencies of position word names in the relational display and attributive display conditions, respectively.

Analysis of variance (ANOVA) was performed for the frequencies of purely position word names in the four display conditions. Taking displays as the unit of analy-
Table 1

Frequency of Various Types for Symbol-Triple Displays, Experiment 1

\begin{tabular}{lcccr}
\hline & \multicolumn{2}{c}{$\begin{array}{c}\text { Relational } \\
\text { Displays }\end{array}$} & \multicolumn{2}{c}{$\begin{array}{c}\text { Attributive } \\
\text { Displays }\end{array}$} \\
\cline { 2 - 5 } & Full & Mixed & HM & HT \\
\hline Position Word Names & 6 & 0 & 1 & 1 \\
Names with Position Words & 8 & 12 & 2 & 1 \\
Total Names Rendered & 52 & 57 & 55 & 56 \\
\hline
\end{tabular}

Note $-H M=$ homogeneous; $H T=$ heterogeneous .

sis, the only significant effect was that of display subtypes within types (full vs. mixed within relational, and homogeneous vs. heterogeneous within attributive) $[F(2,20)=8.08, p<.005]$. This was due to the fact that more position word names occurred in the full relational than in the mixed relational display condition. Taking subjects as the unit of analysis, however, there were no significant effects. Since we wish to generalize to both subjects and displays, we cannot draw any firm conclusions from the analysis.

Osgood (1971) found that people did not often use empty nouns as head constituents for name expressions. $\mathrm{He}$ suggested a weakening of the PCS: Information to distinguish alternatives is attached to ordinary classifying (i.e., nonempty) head nouns. Thus, where Olson's (1970) strategy might predict a name like "the middle one," Osgood's weakened version also allows forms like "the middle window," "the female symbol in the middle," and so on. Frequencies for names with position words, regardless of whether their head nouns were classifying or empty, were also tabulated and are summarized in Table 1. Osgood's criticism of Olson's original statement of the PCS was indeed borne out by the data. The weakened version of the PCS predicts more than $10 \%$ of the name expressions rendered. However, this is still not very good.

Again, however, we contrasted the four stimulus conditions by ANOVA to more sensitively test the PCS predictions. There was a significant main effect of display type (relation vs. attributive), taking displays as the unit of analysis $[F(1,20)=30.17, p<.001]$. This was the only significant effect, however. And in the ANOVA by subject, there were no significant effects whatsoever. Since we wish to generalize to both subject and display populations, we are again in the position of not being able to draw any firm conclusions.

The extreme asymmetry of the frequencies makes it difficult to assess the goodness and ease rating differences. However, the summary statistics are reported in Table 2. On average, position word names were rated as being marginally more difficult to make up and marginally poorer in quality. If anything, these rating differences show that, in addition to not being the naming strategy of choice in this task, the PCS, when it is chosen, produces names that are not remarkably suc- 
Table 2

Ratings of Goodness and Ease for Symbol-Triple Displays, Experiment 1

\begin{tabular}{lcccccc}
\hline & $\begin{array}{c}\text { Relational } \\
\text { Displays }\end{array}$ & & \multicolumn{2}{c}{$\begin{array}{c}\text { Attributive } \\
\text { Displays }\end{array}$} & \\
\cline { 2 - 3 } & Full & Mixed & HM & HT & $\begin{array}{c}\text { Over- } \\
\text { all }\end{array}$ \\
\hline Names with Position Words & 4.18 & 4.83 & 3.00 & 2.00 & 4.48 \\
All Names & 4.32 & 4.80 & 4.14 & 4.83 & 4.55 \\
& & & Ease & & \\
Names with Position Words & 5.55 & 5.50 & 5.00 & 5.00 & 5.46 \\
All Names & 5.12 & 5.72 & 5.18 & 5.92 & 5.48 \\
\hline
\end{tabular}

Note-HM = homogeneous; $H T=$ heterogeneous.

cessful, at least according to the namers themselves.

Notably, perhaps, position word names were rated as better and easier in the relational display conditions than in the attributive display conditions. In this critical contrast, then, we have some suggestion that the PCS is more successful at generating names for relational than for attributive displays. However, here, the frequencies were so small that no strong conclusions can be advanced.

The PCS does predict some of the naming behavior observed in Experiment 1, but actually very little. It was clearly not the strategy of choice, as predicted by Olson (1970). It was not even relatively preferred vis-àvis the relational attributive contrast. Finally, names conforming to the PCS were not rated as being either better or easier than other names. Several "structural" naming strategies predicted more names than the PCS. Indeed, the names generated seemed quite indistinguishable from names rendered for symbols presented without any context set at all (Carroll, Note 2).

For example, $30(13 \%)$ of the names rendered followed the pattern illustrated by "barred triangle": a novel deverbal adjectival shape word followed by another shape word. The referent shape is a triangular shape bisected by a bar. Other examples included the following: "unilegged arc," "crossed circle," "barred square," "dotted bowl," "boxed delta," and "trilegged square." This is a rather specifically structured strategy, and in that sense it is comparable to the PCS, but there were many other less interesting patterns as well: Single word associates, like "fork," "cup," "wheel," "window," and so on, accounted for 51 (22\%) of the names produced. There were also a number of patterns of intermediate complexity.

\section{EXPERIMENT 2}

Since the major result of Experiment 1 was null, a second experiment was conducted to elicit the PCS by explicitly instructing subjects to distinguish target symbols from nontargets. In the specific instructions for the symbol-triples portion of the experiment, the direction "Distinguish the one pointed to from the other two" was substituted for the direction "By what name would you call the one pointed to?" in Experiment 1 . In all other respects, the two experiments were identical.

\section{RESULTS AND DISCUSSION}

Table 3 gives the summary frequencies for Experiment 2. Note that, in sharp contrast to Experiment 1, the PCS was the strategy of choice for relational displays in Experiment 2. Again, we find that the "strong" version of the strategy (that originally proposed by Olson, 1970) predicts a minority of the rendered names, a little more than $20 \%$. However, the weakened PCS (that suggested by Osgood, 1971) predicts more than $50 \%$ of the rendered names. Restricting attention to only the two relational display conditions, the weakened version predicts almost $90 \%$ of the rendered names. Here, the PCS very clearly accounts for the data.

In order to contrast the relational and attributive conditions, we again performed two sets of ANOVAs. First, we analyzed the frequencies of purely position word names. Taking displays as the unit of analysis, there was a main effect of both display type (relational vs. attributive) $[F(1,20)=30.73, p<.001]$ and display subtype within display type $[\mathrm{F}(2,20)=7.97, \mathrm{p}<.005]$. With subjects as the unit of analysis, these effects were also significant [for display type, $F(1,9)=8.54$, $\mathrm{p}<.025$; for display subtype within display type, $\mathrm{F}(2,18)=6.41, \mathrm{p}<.01]$.

In contrast to Experiment 1, we are in a position to draw conclusions. Purely position word names were significantly more frequent in the relational display conditions than in the attributive display conditions. Second, position word names were more frequent in the full relational than in the mixed relational display condition. Both of these results are in full accord with the predictions of the PCS.

The second ANOVA was for the frequencies of names containing some position words. By display, there was a significant main effect of display type $[F(1,20)=$ $3,298.38, p<.001]$, but there was no effect of display subtype within display type. By subject, there was also a significant main effect of display type $[F(1,9)=37.43$, $\mathrm{p}<.001]$ and, again, no significant effect of display subtype within display type. More names with some position words obtained in the relational display condi-

Table 3

Frequency of Various Name Types for Symbol-Triple Displays, Experiment 2

\begin{tabular}{lccrr}
\hline & \multicolumn{2}{c}{$\begin{array}{c}\text { Relational } \\
\text { Displays }\end{array}$} & \multicolumn{2}{c}{$\begin{array}{c}\text { Attributive } \\
\text { Displays }\end{array}$} \\
\cline { 2 - 5 } & Full & Mixed & HM & HT \\
\hline Position Word Names & 23 & 11 & 6 & 5 \\
Names with Position Words & 54 & 53 & 8 & 7 \\
Total Names Rendered & 60 & 59 & 60 & 60 \\
\hline
\end{tabular}

Note-HM = homogeneous; $H T=$ heterogeneous . 
Table 4

Ratings of Goodness and Ease for Symbol-Triple Displays, Experiment 2

\begin{tabular}{lcccccc}
\hline & $\begin{array}{c}\text { Relational } \\
\text { Displays }\end{array}$ & & \multicolumn{2}{c}{$\begin{array}{c}\text { Attributive } \\
\text { Displays }\end{array}$} \\
\cline { 2 - 3 } & Full & Mixed & HM & HT & $\begin{array}{c}\text { Over- } \\
\text { all }\end{array}$ \\
\hline Names with Position Words & 4.87 & 5.21 & 6.25 & 6.29 & 5.19 \\
All Names & 4.67 & 4.95 & 4.90 & 4.98 & 4.88 \\
& & & Ease & & \\
Names with Position Words & 5.65 & 5.77 & 6.88 & 6.71 & 5.84 \\
All Names & 5.42 & 5.57 & 5.22 & 5.65 & 5.46 \\
\hline
\end{tabular}

Note-HM = homogeneous; $H T=$ heterogeneous.

tion than in the attributive condition. This result is, again, fully consistent with the PCS.

Note, though, that in the latter analysis we do not find a significantly greater frequency of names with some position words in the full relational condition than in mixed relational condition. The frequencies of purely position word names support all of the predictions of the PCS. However, the frequencies of names with some position words, those instancing the weakened PCS, only support the overall relational vs. attributive difference and not the full vs. mixed difference.

We again tabulated averages for ratings of goodness and ease. Position word names were rated as being both easier and better than average, in contrast to what was found in Experiment 1. Moreover, the differences were larger than they were in the data of Experiment 1. However, again we must qualify our interpretation of these results, in that the frequencies were so extremely asymmetric. (In Experiment 1, this was because so few names conformed to the PCS; in Experiment 2, it was because almost all of the names for relational displays conformed to the strategy.)

Table 4 also shows that the names with position words were rated as better and easier in the attributive conditions than in the relational conditions. This is the opposite of what was found in Experiment 1, and it is quite at odds with the PCS. Note, though, in Table 3, that the frequencies for names with position words in the attributive conditions were quite small. These contrasts may simply reflect the statistical aberration of small samples. In any case, we can draw no firm conclusions.

In summary, when subjects in the naming experimental situation are given "distinguish" instructions, the (weakened) PCS is clearly the naming strategy of choice-particularly, and as predicted, in the relational display conditions. Also as predicted, the (strong version of the) strategy is more preponderant in the full rela- tional condition than in the mixed relational condition. Finally, names that conform to the PCS seem to be rated as being better names and easier to make up than others.

\section{SUMMARY}

This research begins to clarify the efficient conditions for Olson's (1970) PCS. Clearly, the PCS is not the only strategy for naming and reference. Olson's original characterization of the strategy was very overstated in this regard. The instruction merely to name did not reliably elicit the PCS (Experiment 1 ). In contrast, the instruction to distinguish did elicit names that conform to prediction, although still not without exception (Experiment 2). Finally, where evidence of the PCS appears, it seems predominantly to favor the weakened version suggested by Osgood (1971), not the original statement advance by Olson (1970).

People create names for particular and specific purposes. A simplifying assumption to collapse the dimension of purpose will accordingly be a limited analysis. In fact, just on the basis of the present research, the magnitude of the purpose effect cannot be underestimated: a rather tiny variation in a relatively extensive set of naming instructions created a dramatic change in naming behavior. Olson's (1970) original insight, that referential context controls naming to a great extent, is borne out by the present study, but context is also clearly shown to be an effective variable only through interaction with purpose.

\section{REFERENCE NOTES}

1. Carroll, J. M. Names and naming: An interdisciplinary review: IBM Research Report RC 7370, 1978.

2. Carroll, J. M. Natural strategies in naming. IBM Research Report RC 7533, 1979.

\section{REFERENCES}

Brown, S. Social psychology, New York: Free Press, 1965.

Carroll, J. M. The role of context in creating names. Discourse Processes, 1980, 3, 1-24.

Carroll, J. M. Creating names for things. Journal of Psycholinguistic Research, in press. (a)

Carroll, J. M. Naming and describing in social communication. Language and Speech, in press. (b)

Dreyfus, H. Symbol sourcebook. New York: McGraw-Hill, 1972.

Krauss, R. M., \& Weinheimer, S. Effect of referent similarity and communication mode on verbal encoding. Journal of Verbal Learning and Verbal Behavior, 1967, 6, 359-363.

Olson, D. Language and thought: Aspects of a cognitive theory of semantics. Psychological Review, 1970, 77, 257-273.

OsGood, C. E. Where do sentences come from? In D. Steinberg \& L. Jacobovitz (Eds.), Semantics: An interdisciplinary reader. Cambridge: Cambridge University Press, 1971.

Rosenberg, S., \& Cohen, B. D. Speakers' and listeners' processes in a word-communication task. Science, 1964, 145, 1201-1203.

(Received for publication May 8, 1980.) 